\title{
Editorial
}

Digestion

\section{What Does DNA Methylation Surrogate?}

\author{
Masakazu Toi \\ Department of Surgery, Graduate School of Medicine, Kyoto University, Kyoto, Japan
}

Gastric cancer is a common disease and a major cause of cancer death in some countries in Asia and Europe. It is crucial to prevent disease development and to control disease progression. Many studies have indicated that chronic gastritis caused by Helicobactor pylori is a field where carcinogenesis is likely to occur; however, the details of the process are still largely unknown. Tomomitsu Tahara et al. focused on aberrant DNA methylation, particularly on p14, p16 and p21 genes which are closely involved in the cell growth and cell death of cancer cells, and also looked at the relationship of $H$. pylori infection and cancer histopathology in order to analyze the role of promoter DNA methylation in gastric cancer development. It was found that p14 promoter methylation was significantly associated with $H$. pylori infection and p16 promoter methylation was rather correlated with intestinal metaplasia. Methylation of p21 was uncommon.

According to these results and the relevant literature, the simple question may be raised whether aberrant DNA methylation surrogates in gastric cancer development? Does it surrogate for the progression process in a carci- nogenesis pathway or for the direction to a particular type of gastric cancer? Obviously, it is not easy to classify these characteristics clearly, however, these considerations would help to clarify the function of DNA promoter methylation in gastric cancer carcinogenesis. For instance, it was reported that eradication of $H$. pylori reduced the methylation density in E-cadherin gene and the subsequent neoplastic formation. It is interesting to know what $H$. pylori eradication causes in other types of genes including p14 and p16. A larger study with comprehensive gene sets and with time-course examinations particularly before and after the treatment, such as $H$. pylori eradication or anti-inflammatory therapy, is warranted. Since DNA methylation is engaged in a variety of inflammatory diseases such as ulcerative colitis, Barrett's esophagitis and chronic hepatitis, a model in gastric cancer would be useful for other diseases. In addition, it could be intriguing to look at epidemiological differences in gene promoter methylation, because the H. pylori infection rate in the same lesion differs remarkably between countries .

\section{KARGER \\ Fax +4161306 1234 E-Mail karger@karger.ch} www.karger.com
Masakazu Toi, MD, PhD

Department of Surgery (Breast Surgery)

Graduate School of Medicine, Kyoto University

54 Shogoin-Kawaramachi, Sakyo-ku, Kyoto 606-8507 (Japan)

Tel. +81 375751 3660, Fax +81 375751 3616, E-Mail toi@kuhp.kyoto-u.ac.jp 